KTI/IE Discussion Papers 2005/12

Institute of Economics Hungarian Academy of Sciences

KTI/IE Discussion Papers are circulated to promote discussion and provoque comments. Any references to discussion papers should clearly state that the paper is preliminary. Materials published in this series may subject to further publication.

\title{
The Core Can Be Accessed with a Bounded Number of Blocks
}

\author{
Author: László Á. KÓCZY, Maastricht University, Department of \\ Economics \\ E-mail: l.koczy@algec.unimaas.nl
}

ISSN 1785-377X

ISBN 9639588555

Published by the Institute of Economics Hungarian Academy of Sciences Budapest, 2005 
MT-DP. 2005/12.

KóCZY Á. LÁSZLÓ

A MAG KORLÁTOS SZÁMÚ BLOKKAL IS ELÉRHETÕ

Összefoglaló

Megmutatjuk, hogy ha egy elosztás elérhetõ egy másik elosztásból, az eljutáshoz szükséges blokkok száma korlátos. Ez a korlát csak a vizsgált játékban részt vevõ játékosok számától függ. Nemüres magú játékokra alkalmazva ez azt jelenti, hogy a mag elérhetõ egy korlátos hosszúságú blokksorozattal.

Kulcsszavak: átváltható-hasznosságú játék, mag, indirekt dominancia 


\title{
THE CORE CAN BE ACCESSED WITH A BOUNDED NUMBER OF BLOCKS
}

\author{
LÁSZLÓ Á. KÓCZY
}

\begin{abstract}
We show the existence of an upper bound for the number of blocks required to get from one imputation to another provided that accessibility holds. The bound depends only on the number of players in the TU game considered. For the class of games with non-empty cores this means that the core can be reached via a bounded sequence of blocks.
\end{abstract}

JEL Classification Numbers: C71, C73, C61.

\section{INTRODUCTION}

An international agreement will only be signed if no country or group of countries can do better without it. However, the mere potential for such an agreement does not make it signed. If an initial proposal is more favourable to some, then those countries will not "give in" even if this leads to no agreement; in an election having played hard can easily compensate for an unsigned agreement where blaming other negotiating partners is certainly an easy and hardly refutable explanation. International organisations, such as the UN can be a catalyst by facilitating negotiations. Such an organisation is seen as a player that gets a positive payoff only if an agreement is signed. While it cannot make countries accept less than what they hope to get (under the status quo), it can facilitate the formation of a coalition that blocks the current proposal and makes another one, (temporarily) weakening the bargaining position of the problematic countries thereby making them more inclined to sign an agreement. This way a series of blocks to various proposals can eventually lead to the desired agreement.

Given the existence of a signable agreement, is it possible to manipulate negotiations so that it is actually signed? If yes, can we set deadlines to the negotiations? The first question has already been answered affirmatively $[10,5]$. The present paper answers the second: we show that one can set deadlines and thereby estimate the expected costs of the process already before the negotiations begin.

The core collects agreements that, once proposed, are never abandoned. The question is whether such agreements will ever be proposed. Based on a similar programme by Stearns [12] and Billera [1] for the bargaining set and the kernel, Green [4] and $\mathrm{Wu}[14]$ present transfer schemes that converge to the core. Perry and Reny [6] and Serrano and Vohra [11] defines noncooperative bargaining games that implement core imputations as noncooperative strategy-proof subgame-perfect equilibria. Glycopantis, Muir and Yannelis [2,3] study extensive form implementations of the private core and other solution concepts. Here we take a cooperative approach and use the very same idea that is used to define the core: blocking. Suppose an initial non-core imputation is proposed. Then there exists a sequence

This paper was mostly written at the Catholic University of Leuven. Comments by Luc Lauwers and an anonymous referee have lead to considerable improvements, the remaining errors are of course all mine. I also thank the Catholic University of Leuven and the Netherlands Organisation for Scientific Research (NWO) for financial support. 
of proposals and counter-proposals that eventually leads to the core [10]. Sengupta \& Sengupta [10], however, do not discuss the number of steps required.

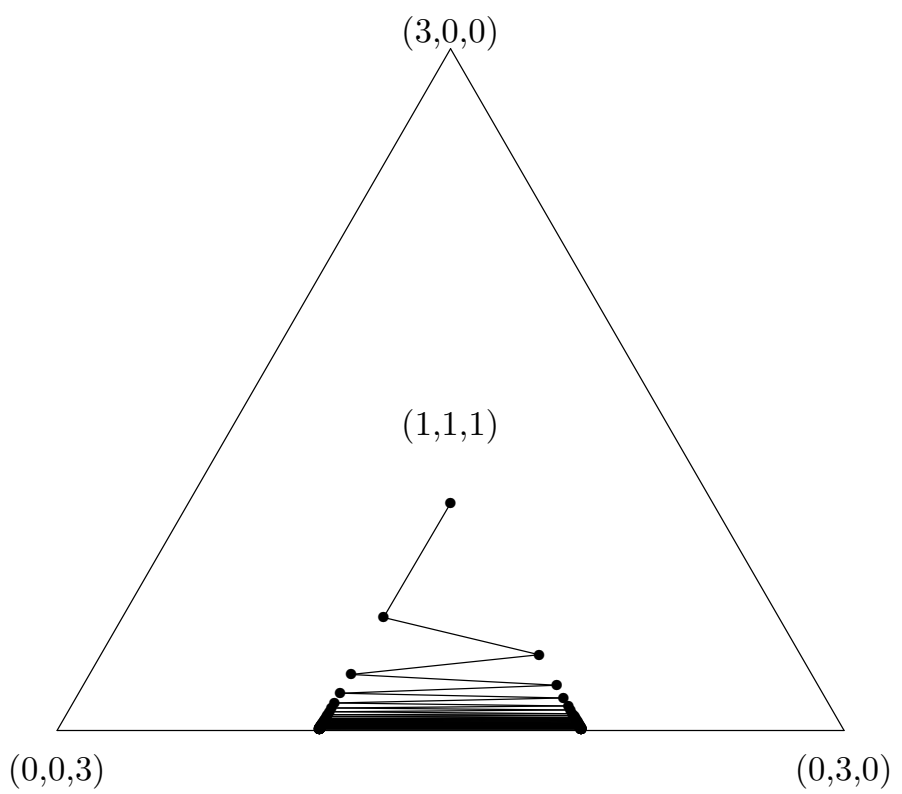

Figure 1. A finite, but unbounded sequence of dominance

Let us illustrate the issue in question by the following 3-player coalitional game where the grand coalition obtains 3 , pairs get 2 , singletons get 0 . The core of this game consists of a single payoff-vector $(1,1,1)$. Now let $a^{k}=\left(\frac{1}{k}, 2-\frac{1}{k}, 1\right)$ and $b^{k}=\left(\frac{1}{k}, 1,2-\frac{1}{k}\right)$, consider an arbitrary positive $m \in \mathbb{N}$, and the following -unnecessarily lengthy- process:

$$
a^{m} \rightarrow b^{m-1} \rightarrow a^{m-2} \rightarrow b^{m-3} \rightarrow \cdots \rightarrow b^{1}=a^{1}=(1,1,1) .
$$

This process terminates in the core in exactly $m-1$ steps. As $m$ is arbitrary the number of steps to reach the core via such a path has no upper bound. The aim of this paper is to show that the core can be reached in a bounded number of steps, moreover, our proof points out where do such inefficient processes make unnecessary detours.

Our result is not specific to the core. We show that if an imputation $b$ can be reached from another imputation $a$ via a path of imputations, then the length of the shortest of such paths is bounded. Since a player would never cooperate to get less than he could by himself, it is realistic to allow only paths via efficient and individually rational allocations, that is, via imputations. Without these restrictions, as in [9] the proof is subject to simplifications.

The structure of the paper is as follows: First we introduce our notation and some terminology. In Section 3 we state our results. The proofs are presented in the Appendices.

\section{Preliminaries}

Let $(N, v)$ be a TU-game with player set $N$, and characteristic function $v$. Subsets of $N$ are coalitions and $v(S)$ is the payoff for coalition $S \subseteq N$. For any pair of vectors $x, y \in \mathbb{R}^{N}$ and set $S \subseteq N$ let $x(S)=\sum_{i \in S} x_{i}$. The restriction of $x$ to $S$ is denoted by $x_{S}$; we write $x_{S} \geq y_{S}$ if $x_{i} \geq y_{i}$ for each $i \in S$, we write $x_{S}>y_{S}$ 
if $x_{S} \geq y_{S}$, but $x_{S} \neq y_{S}$. We denote the vector of stand alone values by $v^{*}$, thus $v^{*}(S)=\sum_{i \in S} v(\{i\})$. For each coalition $S$ let $\bar{S}=N \backslash S$ denote its complementary coalition. Moreover, for the coalition $Q$ let $\bar{v}(Q)=v(N)-v(\bar{Q})$ denote its complementary value, that is, the total payoff left over for the players outside $\bar{Q}$. Note that the complementary value of coalition $S$ is not the value of the complementary coalition $\bar{S} .^{1}$

A payoff-vector $x$ in $\mathbb{R}^{N}$ is an imputation if $x \geq v^{*}$ and $x(N)=v(N)$ : if it is individually rational and efficient. Let $A(N, v)$ denote the set of imputations.

Imputation $y$ directly dominates $x$ via coalition $S$, written $y \succ_{D}^{S} x$ if $y_{S}>x_{S}$ and $y(S)=v(S)$. Then we refer to $S$ as the blocking coalition, and the entire action of obtaining $y$ from $x$ is a block. ${ }^{2}$ The core collects undominated imputations.

Alternative notions of dominance permit $y(S)<v(S)$. Clearly our results will hold if we make dominance a denser relation. Our choice is, however due to our interpretation of dominance. The alternative definition merely compares imputations and is satisfied with the preference for $y$ against $x$, but it does not investigate whether such a transition would actually take place. Our definition is driven by blocks: If a coalition is wants to and can get a higher payoff, it will-myopicallycollect the maximum it can get.

We say that $y$ indirectly dominates $x$ and we write $y \succ_{I} x$ if there exists a finite sequence of imputations $\left\{x^{0}, \ldots, x^{T}\right\}$ and a finite collection of coalitions $\left\{S^{1}, \ldots, S^{T}\right\}$, such that $x=x^{0}, y=x^{T}$, and $x^{t} \succ_{D}^{S^{t}} x^{t-1}$ for all $0<t \leq T$. We call the sequence $\pi=\left\{\left(x^{t}, S^{t}\right)\right\}_{t=1}^{T}$ a (dominance) path, and $T$ its length. The index $t$ is interpreted as time. For convenience we will index paths from 0, and assume that $S^{0}=N$.

In this paper we study such dominance paths and interpret them as processes. The path starts with an initial imputation $x^{0}$. If there exists a coalition $S^{1}$, such that $v\left(S^{1}\right)>x^{0}\left(S^{1}\right)$ then the coalition $S^{1}$ makes a threat of leaving the grand coalition $N$, thereby collecting $v\left(S^{1}\right)$. Due to cohesiveness the remaining players (weakly) prefer to renegotiate and accept a new imputation $x^{1}$, where the demand of coalition $S^{1}$ is satisfied and therefore $x^{1}\left(S^{1}\right)=v\left(S^{1}\right)$, moreover $x^{1}$ directly dominates $x^{0}$ via $S^{1}$. Now $x^{1}$ becomes the status quo, and so on, as long as there exists a coalition who can do better apart than in the current proposal. If no coalition can do better alone, then the imputation belongs to the core.

The existence of a bound is linked to the existence of primitive recursive algorithms: A primitive recursive algorithm is one that can be programmed with "for" loops only [13] and the running time of such a program can be set in advance. See [8] and [7] for more on primitive recursive algorithms.

\section{Results}

Lemma 3.1. For any given game $(N, v)$ there exists an upper bound $M$, such that for all $a, b$ pairs in $A(N, v)$ with $b \succ_{I}$ a there exist a dominance path $\pi$ from $a$ to $b$ with length smaller than $M$.

The proof of Lemma 3.1 is presented in Appendix 4.

\footnotetext{
${ }^{1}$ For instance, in cohesive games we have $v(\bar{S}) \leq \bar{v}(S)$.

${ }^{2}$ The existence of imputation $x$ and coalition $S$ such that $v(S)>x(S)$ do not not generally imply the existence of an imputation $y$ such that $y \succ_{D}^{S} x$. If $v(S)+v^{*}(\bar{S})>v(N)$ such a $y$ would not be both individually rational and efficient for $S$. Since cohesiveness, or even superadditivity are standard assumptions for pure cooperative games, moreover, for our Theorem we consider games with nonempty cores, such a problem cannot arise.
} 
Lemma 3.2 (Theorem in [10]). Let $(N, v)$ be a game with a non-empty core $C(N, v)$. Let a be an imputation outside $C(N, v)$. Then there exists an imputation $c \in C(N, v)$ such that $c \succ_{I} a$.

Theorem 3.3. Let $(N, v)$ be a game with a non-empty core $C(N, v)$. Then there exists an integer $M$ such that for all imputations $a \in A(N, v)$ there exists an imputation $c \in C(N, v)$ and a path $\pi$ from a to $c$ with length smaller than $M$.

Proof. For imputations a in $C(N, v)$ the path $\pi$ is trivial. Otherwise the combination of Lemmata 3.1 and 3.2 gives the desired result.

Lemma 3.4. Let $a$ and $b$ be imputations in $A(N, v)$ with $b \succ_{I} a$. Then there exists a primitive recursive algorithm that defines a path $\pi=\left\{\left(x^{t}, S^{t}\right)\right\}_{t=1}^{T}$ such that $x^{1} \succ_{D}^{S^{1}}$ a, for $1<t \leq T$ we have $x^{t} \succ_{D}^{t} x^{t-1}$, and $x^{T}=b$.

Finally we have the following theorem:

Theorem 3.5. Let $(N, v)$ be a game with a non-empty core $C(N, v)$. Let a be an imputation in $A(N, v)$. Then we show that there exists a primitive recursive algorithm that defines a path $\pi=\left\{\left(x^{t}, S^{t}\right)\right\}_{t=1}^{T}$ such that $x^{1} \succ_{D}^{S^{1}}$ a, for $1<t \leq T$ we have $x^{t} \succ_{D}^{S^{t}} x^{t-1}$, and $x^{T} \in C(N, v)$.

The proofs of Lemma 3.4 and Theorem 3.5 are presented in the Appendices B and C.

\section{Discussion}

The present paper continues the programme initiated by Green [4] and $\mathrm{Wu}$ [14] by establishing a bound on the number of steps needed to reach the core. There are, however some questions that are left open. In this paper our emphasis was on the simplicity of the results rather than on the efficiency of the algorithms. The existence of a bound is encouraging, but it is very likely that the bound can be lowered. While boundedness allows us to define primitive recursive algorithms for the intermediator to design the negotiation process, much lower bounds are required to make the algorithms practical.

\section{REFERENCES}

[1] Louis J. Billera. Global stability in $n$-person games. Transactions of the American Mathematical Society, 172:45-56, 1972.

[2] Dionysius Glycopantis, Allan Muir, and Nicholas C. Yannelis. An extensive form interpretation of the private core. Economic Theory, 18(2):293-319, September 2001.

[3] Dionysius Glycopantis, Allan Muir, and Nicholas C. Yannelis. On extensive form implementation of contracts in differential information economies. Economic Theory, 21(2-3):495-526, March 2003.

[4] Jerry R. Green. The stability of Edgeworth's recontracting process. Econometrica, 42(1):2134, January 1974.

[5] László Á. Kóczy and Luc Lauwers. The coalition structure core is accessible. Games and Economic Behavior, 48:86-93, 2004.

[6] Motty Perry and Philip J. Reny. A noncooperative view of coalition formation and the core. Econometrica, 62(4):795-817, July 1994.

[7] Rózsa Péter. Recursive Functions. Akadémiai, Budapest, New York, London, 1967.

[8] Rózsa Péter. Recursive Functions in Computer Theory. Computers and their Applications. Akadémiai - Ellis Horwood, Budapest - Chichester, 1981.

[9] Abhijit Sengupta and Kunal Sengupta. Viable proposals. International Economic Review, 35(2):347-359, May 1994.

[10] Abhijit Sengupta and Kunal Sengupta. A property of the core. Games and Economic Behavior, 12(2):266-273, 1996.

[11] Roberto Serrano and Rajiv Vohra. Non-cooperative implementation of the core. Social Choice and Welfare, 14(4):513-525, September 1997. 
[12] R. E. Stearns. Convergent transfer schemes for $n$-person games. Transactions of the American Mathematical Society, 134:449-459, December 1968.

[13] Eric W. Weisstein. Primitive recursive functions. Eric Weisstein's World of Mathematics, 2002.

[14] Lilian Shiao-Yen Wu. A dynamic theory for the class of games with nonempty cores. SIAM Journal of Applied Mathematics, 32:328-338, March 1977. 


\section{Appendix A Proof of Lemma 3.1}

A.1. Outline. The proof is by contradiction. We assume that for any number $M_{0}$ there exist imputations $a$ and $b$ such that $b \succ_{I} a$, but the shortest dominance path from $a$ to $b$ is $\pi=\left\{\left(x^{t}, S^{t}\right)\right\}_{t=0}^{T}$ and $T>M_{0}$. We show that if $M_{0} \geq M$ there exist imputations $x^{T_{1}}$ and $x^{T_{2}}$ along the path $\pi$ that are so similar that the subpath from $x^{T_{1}}$ to $x^{T_{2}}$ is essentially a loop that can be removed.

In case $x^{T_{1}}=x^{T_{2}}$ we are done, but in general we have to find a path from $x^{T_{1}}$ to $b$. Note that two imputations are not necessarily connected by a dominance path, but the similarity of $x^{T_{1}}$ and $x^{T_{2}}$ and the existence of a dominance path from $x^{T_{2}}$ to $b$ makes it possible to adapt it. Let $\pi_{3}^{\prime}=\left\{\left(y^{t}, Q^{t}\right)\right\}_{t=0}^{T^{\prime}}$ denote the adapted path. We choose $Q^{t}=S^{T_{2}+t}$ and so $T^{\prime}=T-T_{2}$. Given the blocking coalitions we must define $y^{t}$ such that $(i)$ they are imputations, that is, individually rational and efficient, $(i i)$ the resulting path is a dominance path, $(i i i) \pi_{3}^{\prime}$ indeed ends at $b$. In the following we give an intuition of how we define the imputations $y^{t}$. A formal presentation will follow in Section A.5.2.

Knowing that coalition $Q$ blocks $x$ still leaves us some freedom in choosing the resulting imputation $y$. We only have the following restrictions:

- Players must have at least their stand-alone value, $y \geq v^{*}$.

- Blocking players must get at least as much as before, $y_{Q}>x_{Q}$.

- The total payoff must be efficient $y(N)=v(N)$.

- The payoff for the blocking coalition $Q$ must be efficient, $y(Q)=v(Q)$.

The first two conditions define what we call the subsistence payoff: the bare minimum that has to be distributed. Typically the last two conditions mean that there is more to be distributed. Part of this is distributed within the blocking coalition, and some among the remaining players, that is, the complementary coalition. In distributing this extra we consider future blocks: we give no or minimal extra payoff to those in the the next blocking coalition to further motivate their block. Since the payoff of the players in the complementary coalition $\bar{Q}$ is independent of their previous payoff, they, in essence, lose all they had, including any potential extra payment they had received. Thus, by giving the extra payments to those who do not block in the next round we can waste some payoff that could be inhibitive in later rounds. The problematic cases occur when the entire blocking or complementary coalition blocks, and becomes a faction or cofaction (see Definitions A.1 and A.2) of the next blocking coalition. Then there is no way to get rid of the extra payments. Such cases require special attention. It is comforting though that the (total) payoffs for (co)factions are the same in the adapted as in the original path. It is also true that as soon as such a faction (or at least part of it) is not blocking, the extra payoff can be wasted. Still, most of the definition of similarity is about ensuring that blocks are preserved even if such factions form.

A.2. Example. We illustrate our proof by a simple example with players and a symmetric characteristic function where the payoff of a singleton, a pair, a triple and the grand coalition is $0,0.4,0.7$ and 1 , respectively. The core of this game is nonempty. We consider the randomly generated path in Table 1 that starts from imputation $(0.925,0.025,0.025,0.025)$ and leads to imputation $(0.142,0.3,0.274,0.284)$ that belongs to the core.

After this introduction we can move on to the formal definitions.

A.3. Definitions. The proof requires a number of additional definitions. Let $a$ and $b$ be imputations such that there exists a path $\pi=\left\{\left(x^{t}, S^{t}\right)\right\}_{t=0}^{T}$ leading from $a$ to $b$. As before, $x^{t}$ are the imputations, $S^{t}$ are the blocking coalitions. $S^{0}$ plays 


\begin{tabular}{l|c|c|c}
$t$ & $x^{t}$ & $S^{t}$ & $G^{t}$ \\
\hline 0 & $(0.925,0.025,0.025,0.025)$ & & $\varnothing$ \\
1 & $(0.032,0.374,0.026,0.568)$ & $\{2,3\}$ & $\varnothing$ \\
2 & $(0.08,0.3,0.03,0.59)$ & $\{1,3,4\}$ & $\varnothing$ \\
3 & $(0.28,0.582,0.12,0.018)$ & $\{1,3\}$ & $\varnothing$ \\
4 & $(0.111,0.489,0.341,0.059)$ & $\{3,4\}$ & $\varnothing$ \\
5 & $(0.265,0.373,0.227,0.135)$ & $\{1,4\}$ & $\varnothing$ \\
6 & $(0.141,0.459,0.262,0.138)$ & $\{3,4\}$ & $\varnothing$ \\
7 & $(0.212,0.3,0.316,0.172)$ & $\{1,3,4\}$ & $\varnothing$ \\
8 & $(0.213,0.184,0.416,0.187)$ & $\{1,4\}$ & $\varnothing$ \\
9 & $(0.215,0.185,0.423,0.177)$ & $\{1,2\}$ & $\varnothing$ \\
10 & $(0.329,0.194,0.3,0.177)$ & $\{1,2,4\}$ & $\varnothing$ \\
11 & $(0.535,0.218,0.065,0.182)$ & $\{2,4\}$ & $\varnothing$ \\
12 & $(0.3,0.342,0.092,0.266)$ & $\{2,3,4\}$ & $\varnothing$ \\
13 & $(0.304,0.347,0.096,0.253)$ & $\{1,2\}$ & $\varnothing$ \\
14 & $(0.3,0.348,0.097,0.255)$ & $\{2,3,4\}$ & $\varnothing$ \\
15 & $(0.303,0.214,0.097,0.386)$ & $\{1,3\}$ & $\varnothing$ \\
16 & $(0.3,0.216,0.097,0.387)$ & $\{2,3,4\}$ & $\varnothing$ \\
17 & $(0.379,0.224,0.097,0.3)$ & $\{1,2,3\}$ & $\varnothing$ \\
18 & $(0.04,0.56,0.099,0.301)$ & $\{3,4\}$ & $\varnothing$ \\
19 & $(0.13,0.557,0.27,0.043)$ & $\{1,3\}$ & $\varnothing$ \\
20 & $(0.156,0.444,0.319,0.081)$ & $\{3,4\}$ & $\varnothing$ \\
21 & $(0.171,0.447,0.3,0.082)$ & $\{1,2,4\}$ & $\varnothing$ \\
22 & $(0.085,0.515,0.303,0.097)$ & $\{3,4\}$ & $\varnothing$ \\
23 & $(0.095,0.282,0.305,0.318)$ & $\{1,3\}$ & $\varnothing$ \\
24 & $(0.098,0.283,0.3,0.319)$ & $\{1,3\}$ & $\{1\}$ \\
25 & $(0.104,0.296,0.392,0.208)$ & $\{1,2,4\}$ & $\{1,4\}$ \\
26 & $(0.121,0.357,0.243,0.279)$ & $\{1,2\}$ & $\{1,3,4\}$ \\
27 & $(0.142,0.3,0.274,0.284)$ & $\{1,3,4\}$ & $\{1,2,3,4\}$
\end{tabular}

TABLE 1. Example: payoffs, blocking coalitions and gaining players along a path.

no role, but for completeness we let $S^{0}=N$. The length $T$ is finite; we associate $t$ with time.

Definition A.1 (Faction). If a non-trivial blocking coalition becomes a lasting blocking alliance we refer to it as a faction. Formally, for a given path $\pi$ we say that $F$ is a faction at time $\tau$ if $F=S^{\tau_{0}}$ for some $0<\tau_{0}<\tau$ and $F \subseteq S^{t}$ for all $\tau_{0}<t \leq \tau$, or briefly

$$
F=\bigcap_{t=\tau_{0}}^{\tau} S^{t}=S^{\tau_{0}} .
$$

If a faction contains smaller factions, we consider the largest one.

Definition A.2 (Cofaction). Similarly, if the entire complementary coalition blocks we call it a cofaction. Formally, for a given path $\pi$ we say that $C$ is a cofaction at time $\tau$ if $C=\bar{S}^{\tau_{0}}$ for some $0<\tau_{0}<\tau$ and $C \subseteq S^{t}$ for all $\tau_{0}<t \leq \tau$.

There exists at most one faction, while there may be several cofactions. A blocking or complementary coalition becomes a (co)faction if the coalition is a subset of the next and possibly of subsequent blocking coalitions. Instead of a onetime alliance these players stick together for a longer period and participate in a row of blocks together. Right before becoming a (co)faction $C$ had a total payoff 
of $v(C)$ (or $\bar{v}(C)$ for cofactions) and in the subsequent blocks this total payoff may increase, but cannot decrease. Therefore a (co)faction $C$ must have a total payoff of at least $v(C)$ (or $\bar{v}(C)$ respectively) making a separate treatment necessary. Note that a faction and/or possible cofactions are defined with reference to a period $\tau$ and typically a set of players will not constitute a (co)faction at $\tau^{\prime}>\tau$.

Our example illustrates both very well: At $\tau=7, F=\{3,4\}$ is a faction: it is a blocking coalition at $\tau=6$ and has been a subset of the blocking coalition "ever since". A more complex example were necessary to show how factions can build up and dissolve. Step $\tau=2$ illustrates a cofaction. Since $S^{1}=\{2,3\}$ and its complement blocks in period 2, that is, $S^{2} \supseteq C=\bar{S}^{1}=\{1,4\}$, the coalition $C$ is a cofaction. Note that both the faction and the cofaction preserve the payoffs players have even if it is more than necessary, for the faction this is simply the coalitional payoff, for the cofaction it is the complementary value $\bar{v}(C)=1-0.4=0.6$.

Definition A.3 (Weakest players). Those players in $S \subseteq N$ are the weakest, denoted by $W^{t}(S)$, who are the first not to block, while others in $S$ block. Formally: If there exists a finite $\tau>t$ such that $S \nsubseteq S^{\tau}$ then let $\tau^{*}$ be the minimal such $\tau$ and $W^{t}(S)=S \backslash S^{\tau^{*}}$, otherwise (they are always blocking) $W^{t}(S)=S$.

If $S$ is the blocking coalition, the weakest players are the "weakest link" in it: in the example at $t=9$ and for $S=\{1,2\}$ the weakest link is $\{1\}$ as 1 will block only until $t=10$, while 2 blocks until $t=14$.

If $S$ is a complementary coalition the weakest players are those that do not block in the next step. If such players do not exist, a cofaction is formed, and the weakest players are those dropping out first. We see this at $t=1$ with $S=\{1,4\}$ or at $t=20$ with $S=\{1,2\}$. While $W^{1}(\{1,4\})=\{4\}$, we have $W^{20}(\{1,2\})=\{1,2\}$, that is, players in $S$ may be equally weak.

Definition A.4 (Subsistence level). Given a dominance path $\pi=\left\{\left(x^{t}, S^{t}\right)\right\}_{t=0}^{T}$ we define the subsistence level $s^{t} \in \mathbb{R}^{N}$ for all $t>0$ as the vector of minimal payoffs that allows $x^{t}$ to be individually rational and that makes the block $S^{t}$ profitable. Therefore:

$$
s_{i}^{t}= \begin{cases}x_{i}^{t-1} & \text { if } i \in S^{t} \\ v(\{i\}) & \text { otherwise }\end{cases}
$$

Definition A.5 (Surplus vector). For given $z \in \mathbb{R}, S \subseteq N$ and time $t$ let $D^{t}(S, z) \subseteq$ $\mathbb{R}^{N}$ denote the set of vectors $d>0$ with $d(N)=d\left(W^{t}(S)\right)=z$, that is, vectors $d$ that share $z$ among the weakest players. Surplus vectors are then the elements of $\tilde{D}^{t}(S, z)=\arg \min _{d \in D^{t}(S, z)} \max _{i}\left\{b_{i}-\left(s_{i}^{t}+d_{i}\right)\right\}$. Let $\tilde{d}^{t}(S, z) \in \tilde{D}^{t}(S, z)$.

The set $\tilde{D}^{t}(S, z)$ is well defined, in fact $\tilde{d}^{t}(S, z)$ is unique. ${ }^{3}$ The surplus vector reduces the "distance" from the final imputation $b$ while making sure that gaining players (see Definition A.8) never exceed their final payoff. It will be used to allocate extra gains beyond the subsistence level and hence the name.

Definition A.6 (Concatenation). If $\pi_{1}=\left\{\left(x_{1}^{t}, S_{1}^{t}\right)\right\}_{t=0}^{T_{1}}$ and $\pi_{2}=\left\{\left(x_{2}^{t}, S_{2}^{t}\right)\right\}_{t=0}^{T_{2}}$ are paths such that $x_{2}^{0}=x_{1}^{T_{1}}$, we can define their concatenation as

$$
\pi_{1} \wedge \pi_{2}=\left\{\left(x_{1}^{0}, S_{1}^{0}\right),\left(x_{1}^{1}, S_{1}^{1}\right), \ldots,\left(x_{1}^{T_{1}}, S_{1}^{T_{1}}\right),\left(x_{2}^{1}, S_{2}^{1}\right), \ldots,\left(x_{2}^{T_{2}}, S_{2}^{T_{2}}\right)\right\} .
$$

\footnotetext{
${ }^{3}$ We can construct $\tilde{d}^{t}(S, z)$ as follows: Per definition $\tilde{d}^{t}(S, z)_{i}=0$ if $i$ is not among the weakest players in $S$. So we focus on the weakest players in $S$. Distribute $z$ gradually, always increasing $\tilde{d}^{t}(S, z)_{i}$, where $i$ maximises $b_{i}-\left(s_{i}^{t}+\tilde{d}^{t}(S, z)_{i}\right)$. Clearly, this decreases the maximum and bring us closer to the minimum. When $z$ is consumed we are done.
} 
A.4. Defining the bound $M$. We define a classification of imputations. The number of classes is $M$, that is to be the upper bound in Lemma 3.1.

Definition A.7 (Similar imputations). Imputations $x$ and $y$ are similar if for all quadruples of mutually disjoint coalitions $Q_{F}, Q_{C}, Q_{S}$ and $Q_{I}$ with $Q_{F} \cup Q_{C} \cup$ $Q_{S} \cup Q_{I}=Q \subsetneq N$ and $Q_{I} \neq \varnothing$, and all partitions $\mathcal{Q}_{C}$ of $Q_{C}$ we have

$$
\begin{aligned}
& v\left(Q_{F}\right)+\sum_{C \in \mathcal{Q}_{C}} \bar{v}(C)+v^{*}\left(Q_{S}\right)+x\left(Q_{I}\right) \geq v(Q) \quad \text { if and only if } \\
& v\left(Q_{F}\right)+\sum_{C \in \mathcal{Q}_{C}} \bar{v}(C)+v^{*}\left(Q_{S}\right)+y\left(Q_{I}\right) \geq v(Q) .
\end{aligned}
$$

Similar imputations are safe against the same blocks (that is $Q_{F}=Q_{C}=Q_{S}=$ $\varnothing)$, even after certain modifications, where the initial differences are limited to a subset of the players. While the particular construction will be understood in Proposition A.10 the motivation is clear: if coalition $Q_{F}$ has its coalitional payoff, all coalitions $C \in \mathcal{Q}_{C}$ have their complementary value, players in $Q_{S}$ their individually rational value instead of the original payoff, then imputations $x$ and $y$ must still neither or both be dominated via coalition $Q$.

In our example $x^{3}$ and $x^{19}$ are similar. In order to establish similarity one has to check all, in this case a total of 47 different conditions. For instance, consider $Q=\{1,2\}$, such that $1 \in Q_{C}$ and $2 \in Q_{I}$. Then the condition becomes $\bar{v}(\{1\})+$ $x_{2} \geq v(\{1,2\})$ if and only if $\bar{v}(\{1\})+y_{2} \geq v(\{1,2\})$. This condition states that if there is a path from $x$, such that at period $\tau$ the coalition $\{1,2\}$ blocks, moreover, player 1 constitutes a cofaction and player 2 has a history of continuous blocking from the start, then there is a path from $y$, where coalition $\{1,2\}$ also blocks, and vice versa.

Now consider a path $\pi=\left\{\left(x^{t}, S^{t}\right)\right\}_{t=0}^{T}$. Let $G^{t}=\bigcup_{\tau=t+1}^{T} S^{\tau}$ denote the set of gaining players: players who are sure not to lose, but only to gain in the remaining $\tau>t$ part of the path. Such players require special attention as along the modified path their payoff can only be increased, but not lowered. Observe that the set of gaining players monotonically increases, and within one path there are $n+1$ possible different sets, with $G^{t}=N$ only possible when $t=T$.

Definition A.8. Imputations that are similar and have the same set of gaining players belong to the same class.

Note that having the a certain set of gaining players is a path dependent property, however the number of pigeon-holes needed is the same for all paths. Since Equation A.2 really creates partitions of $N$ we have a finite number of classes. Let $M$ denote this number. If the number of imputations in $\pi$ exceeds $M$ we are guaranteed to find $T_{1}<T_{2}$ such that the imputations $x^{T_{1}}$ and $x^{T_{2}}$ are similar.

In practice even relatively short paths, such as our example will already have similar imputations. As we have already mentioned, in our example $x^{3}$ and $x^{19}$ are similar, moreover they have the same (empty) set of gaining players and therefore they belong to the same class.

A.5. Creating a shorter path. Let $a, b \in A(N, v)$ be such that $b \succ_{D} a$ and $\pi=\left\{\left(x^{t}, S^{t}\right)\right\}_{t=0}^{T}$ be a shortest path from $a$ to $b$. Assume that $\pi$ has a length exceeding $M$. We construct a shorter path by modifying it: we seek two similar imputations $x^{T_{1}}$ and $x^{T_{2}}$, remove the subpath $\pi_{2}$ connecting them, modify the tail $\pi_{3}$ to get $\pi_{3}^{\prime}$ and reattach it to the head $\pi_{1}$. The resulting path $\pi^{\prime}=\pi_{1} \wedge \pi_{3}^{\prime}$ is shorter than $\pi$, giving the desired contradiction. 


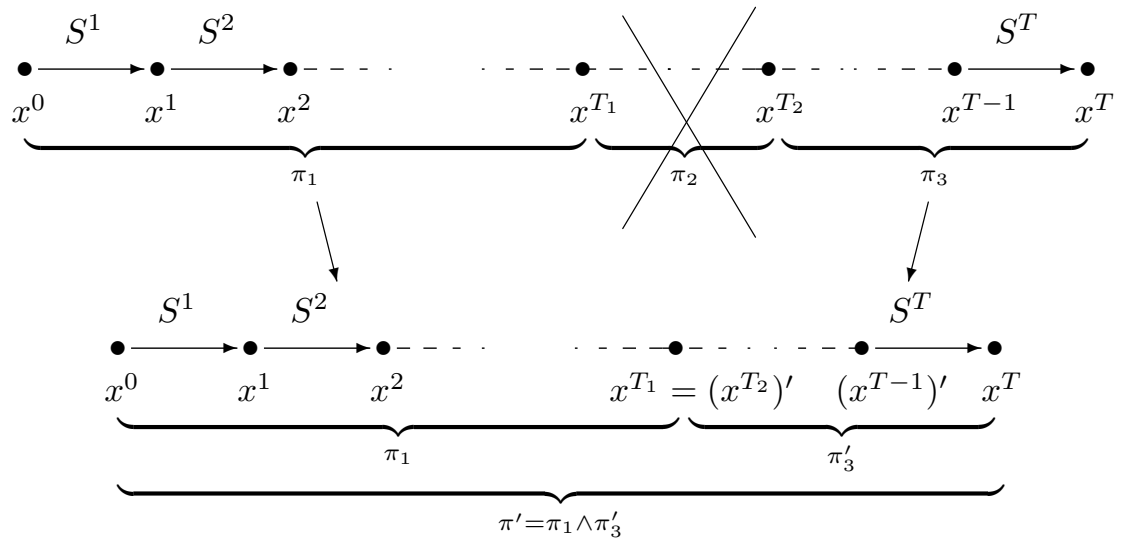

FIGURE 2. Schematic picture of the proof.

A.5.1. Trisection of path $\pi$. As a first step in defining the new path we look for similar imputations along $\pi$. By the assumption that $T>M$ such imputations exist. Let us denote them by $x^{T_{1}}$ and $x^{T_{2}}$. The imputations $x^{T_{1}}$ and $x^{T_{2}}$ cut $\pi$ into 3 subpaths $\pi_{1}, \pi_{2}$, and $\pi_{3}$ so that $\pi=\pi_{1} \wedge \pi_{2} \wedge \pi_{3}$.

A.5.2. A similar path: $\pi_{3}^{\prime}$. Our aim is to find the path $\pi_{3}^{\prime}$ from $x^{T_{1}}$ to $b$. While two imputations need not be connected by a dominance path in general, the existence of a dominance path from $x^{T_{2}}$ to $b$ and the similarity of $x^{T_{1}}$ and $x^{T_{2}}$ makes the definition possible in this case. We make use of a particular construction that uses the same sequence of blocks.

In order to avoid cumbersome notation we write $Q^{t}$ instead of $\left(S^{T_{2}+t}\right)^{\prime}=S^{T_{2}+t}$ and $y^{t}$ instead of $\left(x^{T_{2}+t}\right)^{\prime}$ for all $0 \leq t \leq T-T_{2}$. Therefore the new path is $\pi_{3}^{\prime}=\left\{\left(y^{t}, Q^{t}\right)\right\}_{t=0}^{T^{\prime}}$, where $T^{\prime}=T-T_{2}$.

Then we define path $\pi_{3}^{\prime}=\left\{\left(y^{t}, Q^{t}\right)\right\}_{t=0}^{T^{\prime}}$ by

$$
Q^{t}=S^{T_{2}+t} \quad \text { and } \quad y^{t}= \begin{cases}x^{T_{1}} & \text { if } t=0 \text { and } \\ s^{t}+\delta^{t}+\epsilon^{t} & \text { for } 0<t \leq T^{\prime},\end{cases}
$$

where $s^{t}$ is the subsistence payoff and $\delta^{t}$ and $\epsilon^{t}$ are the surplus vectors

$$
\begin{aligned}
\delta^{t} & =\tilde{d}^{t}\left(S^{t}, v\left(S^{t}\right)-s^{t}\left(S^{t}\right)\right) \quad \text { and } \\
\epsilon^{t} & =\tilde{d}^{t}\left(\bar{S} t, \bar{v}\left(\bar{S}^{t}\right)-s^{t}\left(\bar{S}^{t}\right)\right) .
\end{aligned}
$$

What remains is to show that $\pi_{3}^{\prime}$ is a dominance path from $x^{T_{1}}$ to $x^{T}$.

A.6. An example. Let us first apply our method to our example.

We have already found that imputations $x^{3}$ and $x^{19}$ are similar and therefore they can be used to create a shorter path. This shorter path consists of $x^{0}, x^{1}, x^{2}, x^{3}$ and a modified versions of $x^{20}, \ldots, x^{27}$ as defined by Equation A.3: we use the same sequence of blocking coalitions. These determine the weakest players in each blocking coalition as well as in its complement. Since the future of each of the players is typically different, the set of weakest players is mostly a set containing a single element: the surplus vectors assign the surplus to this player and then finding the next imputation is straightforward. To this only mputations $y^{1}, y^{2}$ and $y^{7}$ are exceptions. In the first two we see players 1 and 2 forming a cofaction for a single round and then be blocked by 3 and 4 . As a result the distribution of the complementary payoff is actually irrelevant. Imputation $y^{7}$ is more interesting: all blocking players age gaining players and so there will be no further chance to decrease their payoffs. The definition of surplus vectors, however ensures that no payoff runs over. 


\begin{tabular}{|c|c|c|c|c|c|c|c|c|}
\hline$t$ & \multicolumn{4}{|c|}{$y^{t}$} & $Q_{F}^{t}$ & $Q_{C}^{t}$ & $Q_{S}^{t}$ & $Q_{I}^{t}$ \\
\hline 0 & $\begin{array}{l}0.925, \\
(0.32, \\
(0.08, \\
(0.280\end{array}$ & $\begin{array}{l}0.25, \\
0.374, \\
0.3, \\
0.582,\end{array}$ & $\begin{array}{l}0.25, \\
0.26, \\
0.030, \\
0.120\end{array}$ & $\begin{array}{l}0.25) \\
0.568) \\
0.590) \\
0.018)\end{array}$ & & & & \\
\hline 1 & $(0.221$ & 0.379 & 0.382 , & $0.018)$ & $\varnothing$ & $\varnothing$ & $\varnothing$ & $\{3,4\}$ \\
\hline 2 & $(0.262)$ & 0.42 & 0.3 & $0.018)$ & $\varnothing$ & $\{1,2\}$ & $\varnothing$ & $\{4\}$ \\
\hline 3 & $(0$, & 0.6 , & $0.3)$ & $0.1)$ & $\varnothing$ & $\{3\}$ & $\varnothing$ & $\{4\}$ \\
\hline 4 & (0, & 0 , & 0.4 & $0.6)$ & $\varnothing$ & $\{3\}$ & $\{1\}$ & $\varnothing$ \\
\hline 5 & $(\mathbf{0}$ & $\mathbf{0}$ & 0.3 & $0.7)$ & $\varnothing$ & $\{2,4\}$ & $\{1\}$ & $\varnothing$ \\
\hline 6 & $(0$ & 0.4 & 0.6 & 0.) & $\varnothing$ & $\varnothing$ & $\{1,2\}$ & $\varnothing$ \\
\hline 7 & (0.129), & 0.6 & 0 , & $0.271)$ & $\varnothing$ & $\varnothing$ & $\{1,4\}$ & $\varnothing$ \\
\hline 8 & (0.142, & 0.3 & 0.274 & $0.284)$ & $\{1,4\}$ & $\varnothing$ & $\{3\}$ & $\varnothing$ \\
\hline
\end{tabular}

TABLE 2. The (shorter) path generated by the algorithm. Boldface indicates blocking coalitions $Q^{t}$. The right hand side refers to Proposition A.10 on page 11.

Our example also illustrates how easy it actually is to generate a similar path that 'works'.

A.6.1. The path $\pi_{3}^{\prime}$ is a dominance path. We show that the path $\pi_{3}^{\prime}$ generated in Subsection A.5.2 is a dominance path from $x^{T_{1}}$ to $x^{T}$. To do so we show that

- All elements of $\pi_{3}^{\prime}$ are imputations (Proposition A.9).

- The path is a dominance path, that is, an imputation is directly dominated by the next imputation (Propositions A.10, A.11, and A.12).

- Finally we need to check that the path is indeed from $x^{T_{1}}$ to $x^{T}$. While the first holds per definition, the second requires an additional result, Proposition A.13.

All propositions, except the last one will be shown by induction over $t$.

Proposition A.9. If $0 \leq t \leq T^{\prime}$, the vector $y^{t}$ is an imputation.

The second proposition as about the anatomy of a blocking coalition.

Proposition A.10. If $0 \leq t<T^{\prime}$ the blocking coalition $Q^{t+1}$ partitions into:

$Q_{F}::$ Players belonging to a faction.

$Q_{C}:$ : Players belonging to a cofaction.

$Q_{S}:$ : Players whose fellow members from their last complementary coalition have some been non-blocking since then.

$Q_{I}::$ Players who never belonged to the complementary coalition.

Moreover, players $i$ in $Q_{S}$ have $y_{i}^{t}=v(\{i\})$, players $j$ in $Q_{I}$ their original payoff $y_{j}^{t}=y_{j}^{0}$.

Proposition A.11. If $0 \leq t \leq T^{\prime}$ the block by $Q^{t+1}$ is profitable: $v\left(Q^{t+1}\right)>$ $y^{t}\left(Q^{t+1}\right)$.

Proposition A.12. If $0<t \leq T^{\prime}$ the imputation $y^{t}$ directly dominates $y^{t-1}$ by coalition $Q^{t}$, that is $y^{t} \succ_{D}^{Q^{t}} y^{t-1}$.

Proposition A.13. The imputations $y^{T^{\prime}}$ and $x^{T}=b$ coincide.

The two less intuitive results are Propositions A.10 and A.13. While the latter is, as we will see, a result of certain properties of the surplus vector we illustrate the first by our example. 
A blocking coalition will contain a faction if it contains the previous blocking coalition (which, itself, is then a faction), as in $y^{8}$. It will contain a cofaction if it contains the entire complementary coalition as in $y^{2}, y^{3}$ or $y^{5}$. These can also survive 'nested' in a larger faction: in $y^{4}$ the blocking coalition contains player 3 that constitutes a cofaction since $y^{2}$. A player belongs to $Q_{S}$ if it comes with a fraction of the complementary coalition, possibly via a cofaction. An example of the latter is player 2 in $y^{6}$ : it is a fraction of the complementary coalition $\bar{Q}^{4}$. Finally, player 4 belongs to $Q_{I}$ for a while as it has a winning streak since the original payoff. Note that if players 3 and 4 would belong to the blocking coalition after $y^{t}$, they would belong to $Q_{F}$ as they would have a surplus they have to carry. If, after that, only one of them, say 4 would be blocking then 4 would again belong to $Q_{I}$, while 3 would never again.

Now we proceed to the proof itself.

Step 1. Trivial case, $t=0$.

Proposition A.9. Since $x^{T_{1}}$ is an imputation $y^{0}$ is also an imputation.

Proposition A.10. Trivially all $i \in Q^{1}$ satisfy $i \in Q_{I}$.

Proposition A.11. Since $y^{0}=x^{T_{1}}$ and $x^{T_{2}}$ are similar, and $v\left(Q^{1}\right)>x^{T_{2}}\left(Q^{1}\right)$, the block by $Q^{1}$ is profitable, that is, $v\left(Q^{1}\right)>y^{0}\left(Q^{1}\right)$.

Proposition A.12 is an empty statement here.

Step 2. Inductive assumption.

We assume that for each $0<\tau<t$ the imputation $y^{\tau}$ has already been defined, and we have shown that Propositions A.9, A.10, A.11 and A.12 hold.

Step 3. Inductive step.

Proposition A.9. By Proposition A.9 for $y^{t-1}$, by the definition of subsistence levels and since $\delta^{t}>0, \epsilon^{t}>0$ the vector $y^{t}$ is individually rational. By construction $y^{t}(N)=s^{t}(N)+\delta^{t}(N)+\epsilon^{t}(N)=v(N)$ so efficiency is also satisfied.

Proposition A.10. We consider 3 cases $^{4}$ based on the type of the block $Q^{t+1}$.

(1) $Q^{t} \subseteq Q^{t+1}$. Then $Q^{t}$ is a faction and hence $Q_{F}=Q^{t}$. Since $Q^{t+1} \neq N$, $\bar{Q}^{t} \not \subset Q^{t+1}$. Then for each $i \in \bar{Q}^{t} \cap Q^{t+1}$ we have $s_{i}^{t}=v(\{i\})$ and $\delta_{i}^{t}=\epsilon_{i}^{t}=$ 0 , since $i \notin Q^{t}$, and $i \notin W^{t}\left(\bar{Q}^{t}\right)$. Thus $y_{i}^{t}=v(\{i\})$ and $Q_{S}=Q^{t+1} \backslash Q^{t}$. See also $t=7$ in the example: there $Q_{F}^{8}=\{1,4\}$ and $Q_{S}^{8}=\{3\}$.

(2) $\bar{Q}^{t} \subseteq Q^{t+1}$. Then $\bar{Q}^{t}$ acts as a cofaction and hence $\bar{Q}^{t} \subseteq Q_{C}$. As $Q^{t+1} \neq N$ and $Q^{t} \not \subset Q^{t+1}$ we cannot have $Q^{t}$ as a faction. For each $i \in Q^{t+1} \backslash \bar{Q}^{t}$ by definition we have $s_{i}^{t}=y_{i}^{t-1}$, moreover $\delta_{i}^{t}=\epsilon_{i}^{t}=0$ since $i \notin \bar{Q}^{t}$ and $i \notin W^{t}\left(Q^{t}\right)$ : thus $y_{i}^{t}=y_{i}^{t-1}$.

We apply the inductive assumption for players in $Q^{t}$.

(a) Players in $Q_{S}^{t} \cap Q^{t+1}$ with their initial or individually rational payoffs belong to $Q_{S}^{t+1}$ as well.

(b) With the exception of $\bar{Q}^{t}$, cofactions in $Q_{C}^{t+1}$ are part of $Q_{C}$.

(c) If there is a faction that survives it becomes set $Q_{F}$.

(d) The interesting players are those that are members of a faction or a cofaction that just broke up. In either case, by construction, any surplus gained was given to the weakest players that are not in $Q^{t+1}$. Hence, in the latter case players have their stand alone payoff and belong

\footnotetext{
${ }^{4}$ The fourth case, $Q^{t} \subseteq Q^{t+1}$ and $\bar{Q}^{t} \subseteq Q^{t+1}$ would imply $N \subseteq Q^{t+1}$ and hence $N=Q^{t+1}$, which does not make sense
} 
to $Q_{S}$. In the former case we get the pre-deviation payoffs of players participating in an earlier deviation. By the induction hypothesis Proposition A.11 holds for this coalition as well, and we can repeat our arguments. As a blocking coalition must, by definition, strictly contain any possible (co)faction, we gradually assign all players to one of the sets except potentially a set $Q_{I}$ of players that are winning from the start, but, by construction, these have their initial payoffs.

(3) Finally we consider the case when $Q^{t} \nsubseteq Q^{t+1}$ and $\bar{Q}^{t} \nsubseteq Q^{t+1}$. First let $i \in \bar{Q}^{t} \cap Q^{t+1}$. By the same argument as in Case 1 , we have $y_{i}^{t}=v(\{i\})$ and $\left(Q^{t+1} \backslash Q^{t}\right) \subseteq Q_{S}$. Now let $j \in Q^{t} \cap Q^{t+1}$. By the same argument as in Case 2, $y_{j}^{t}=y_{j}^{\bar{t}-1}$ and since $j \in Q^{t}$, Proposition A.10 for $y^{t-1}$ implies it for $y^{t}$.

Proposition A.11. We use the notation of Proposition A.10.

Let us partition players in $Q^{t+1}$ according to Proposition A.10. We have

$$
y^{t}\left(Q^{t+1}\right)=v\left(Q_{F}\right)+\sum_{C \in \mathcal{Q}_{C}} \bar{v}(C)+v^{*}\left(Q_{S}\right)+y_{0}\left(Q_{I}\right),
$$

where $\mathcal{Q}_{C}$ is the collection of cofactions in $Q_{C}$. Note that $Q_{C}$ and/or $Q_{S}$ may be empty.

Now consider the corresponding payoffs in path $\pi$. The players are involved in blocks by the same coalitions and hence the same partition of $Q^{t+1}$ forms. However, in this "organic" path extra payoffs are distributed in an uncontrolled way so that the payoffs of not only the weakest players can increase. Therefore players in the sets $Q_{F}, Q_{I}, Q_{S}$ and $Q_{C}$ can collect (but not lose!) additional payoff in addition to the payoffs characteristic for the group. Since the block by $S^{t+T_{2}}=Q^{t}$ is profitable

$$
v\left(Q^{t+1}\right)>x^{t+T_{2}}\left(Q^{t+1}\right) \geq v\left(Q_{F}\right)+\sum_{C \in \mathcal{Q}_{C}} \bar{v}(C)+v^{*}\left(Q_{S}\right)+x^{T_{2}}\left(Q_{I}\right) .
$$

Since $x^{T_{2}}$ and $y^{0}=x^{T_{1}}$ are similar, Equations A.6 and A.7 imply $v\left(Q^{t+1}\right)>$ $y^{t}\left(Q^{t+1}\right)$.

Now assume that $Q^{t+1}$ does not contain players who have their initial payoffs at $t$, but possibly a composite player. This modifies Equation A.6 as follows:

$$
y^{t}\left(Q^{t+1}\right)=\sum_{C \in \mathcal{R}_{C}} \bar{v}(C)+v^{*}\left(R_{S}\right)+v\left(R_{O}\right) .
$$

As before, in the other path blocks can lead to gains, hence:

$$
v\left(Q^{t+1}\right)>x_{3}^{t}\left(Q^{t+1}\right) \geq \sum_{C \in \mathcal{R}_{C}} \bar{v}(C)+v^{*}\left(R_{S}\right)+v\left(R_{O}\right) .
$$

Combining this equation with Equation A.8 we get $v\left(Q^{t+1}\right)>y^{t}\left(Q^{t+1}\right)$ as required.

Proposition A.12. For $i \in Q^{t}$ the subsistence level $s_{i}^{t}=y_{i}^{t-1}$ already guarantees the level of pre-block payoffs. By the weaker Proposition A.11 for $y^{t-1}$ blocks are profitable and so the equaliser function $\delta^{t}$ shares a strictly positive amount, $\delta^{t}\left(Q^{t}\right)>0$ among the members of $Q^{t}$ and, per construction, $\delta_{i}^{t} \geq 0$. Since $\epsilon_{i}^{t}=0$, we conclude $y_{Q^{t}}^{t}=s_{Q^{t}}^{t}+\delta_{Q^{t}}^{t}+\epsilon_{Q^{t}}^{t}>y_{Q^{t}}^{t}$.

Proposition A.13. What remains to prove is that the newly constructed path not only starts, but also arrives at the right imputation.

Due to the particular construction of $\pi_{3}^{\prime}$, the payoff for players who are not dominating is defined without reference to their previous payoffs. Hence a player 
may accumulate however high payoff, it will also lose it unless it belongs to the gaining set.

Now we use the fact that $x^{T_{1}}$ and $x^{T_{2}}$ were selected in part because they have the same gaining sets, $G^{T_{1}}=G^{T_{2}}$ of players so that $x_{i}^{T_{1}} \leq x_{i}^{T_{2}}$ for all $i \in G^{T_{1}}$. The definition of surplus vectors ensures that they are always the weakest players who get the profit of the deviation, and these are typically not the gaining players. However, if the gaining players are also the weakest (they are clearly the "strongest", but the two may coincide) the payoff equalising vectors ensure that none of the gaining players is assigned a payoff higher than the respective payoff in $b=x^{T}$. Since $G^{T^{\prime}-1}=N$, by efficiency they must also receive exactly the payoff at $b$.

\section{Appendix B Proof of Lemma 3.4}

Sengupta and Sengupta [10] define a dominance path from any outcome to the core, but since their algorithm is not primitive recursive their result can be used here but to the extent of existence. In Section A.5.2 we have constructed a path of length at most $M$ relying only on the sequence of blocking coalitions. Since the number of coalitions is finite, so is the number of possible sequences of length at most $M$. Then our algorithm can be defined as follows.

(1) Generate all coalition sequences $\left\{Q^{t}\right\}_{y=1}^{T}$ of length at most $M$.

(2) For all such blocking sequences do the following loop:

(a) Attempt to generate $\left\{y^{t}\right\}_{y=1}^{T}$ using Equation A.3.

(b) If generation is successful then per construction it is a bounded sequential domination path. Exit loop.

(c) Take the next blocking sequence.

(3) Stop.

Since $a \succ_{I} b$, by Lemma 3.1 there exists a path $\pi_{3}^{\prime}=\left\{\left(\tilde{y}^{t}, \tilde{Q}^{t}\right)\right\}_{y=1}^{T^{\prime}}$. The algorithm goes through all coalition sequences including $\left\{\tilde{Q}^{t}\right\}_{y=1}^{T^{\prime}}$ so it finds it, unless it finds another suitable sequence and terminates sooner. The algorithm is primitive recursive by construction.

\section{Appendix C Proof of Theorem 3.5}

We begin by the following lemma.

Lemma C.14. Let $a \in A(N, v), c \in C(N, v)$ and $\left\{Q^{t}\right\}_{t=1}^{T}$ a sequence of coalitions. Then there exists a dominance path $\left\{\left(y^{t}, Q^{t}\right)\right\}_{t=0}^{T}$ from a to $c$ if and only if

(1) for all $i \in \bigcap_{t=1}^{T} Q^{t}$ we have $b_{i} \geq c_{i}$,

(2) $v\left(Q^{T}\right)=c\left(Q^{T}\right)$, moreover

(3) if $\exists 0<t<T$ such that $\bar{Q}^{t} \subseteq Q^{t^{\prime}}$ for all $t^{\prime}>t$ then $c\left(Q^{t}\right)=v\left(Q^{t}\right)$.

Proof. The if-part holds by the construction of $\left\{y^{t}\right\}_{t=0}^{T}$ as given in Section A.5.2. For the only-if part we assume that the whole dominance path is generated and $y^{T}=$ $b$. For those in the complementary coalition $\bar{Q}^{T}$ the latter is granted per definition of $\epsilon^{T^{\prime}}$. We focus on the players in the last blocking coalition $Q^{T}$. Property $B^{t}$ in the proof of Lemma 3.1 partitioned the members of $Q^{T}$ into four sets.

- If $i \in Q_{S}$ then $y_{i}^{T-1}=v(\{i\})$. Since $c$ is an imputation $c_{i} \geq y_{i}^{T-1}$ must hold.

- $Q_{F}$ obtained a payoff of $v\left(Q_{F}\right)$ when it was blocking and has since then only increased its payoff. However $c(S) \geq v(S)$ holds for all coalitions $S$. 
- For $Q_{C}$ we require $c\left(Q_{C}\right) \geq y^{T-1}\left(Q_{C}\right) \geq \bar{v}\left(\bar{Q}_{C}\right)$. On the other hand, since $c$ is a core imputation $c\left(\bar{Q}_{C}\right) \geq v\left(\bar{Q}_{C}\right)$ so $c\left(Q_{C}\right) \leq \bar{v}\left(\bar{Q}_{C}\right)$. Hence $c\left(Q_{C}\right)=\bar{v}\left(\bar{Q}_{C}\right)$ or alternatively $c\left(\bar{Q}_{C}\right)=v\left(\bar{Q}_{C}\right)$. Since $Q_{C}=\bar{Q}^{t}$ for some $t$ such that $\bar{Q}^{t} \subseteq Q^{t^{\prime}}$ for all $t^{\prime}>t$, we must have $c\left(Q^{t}\right)=v\left(Q^{t}\right)$.

- Finally we consider $Q_{I}$ that contains players with their initial payoffs $y_{i}^{0}$. Such players have a winning streak from the beginning to the end so their payoff monotone increases. Hence for all such $i$ the relation $a_{i} \leq c_{i}$ must hold.

So we conclude that all conditions of Lemma C.14 are necessary for profitable blocks along $\left\{\left(y^{t}, Q^{t}\right)\right\}_{t=0}^{T}$.

Proof of Theorem 3.5. If we knew the core imputation $c$ that is accessible from $a$ then we could simply use Lemma 3.4 to prove the theorem. But not only that we do not know such a $c$, but there are in general a continuum of imputations in $C(N, v)$ all of which are potential candidates, so checking all of them one-by-one is not an option. However given $a$ and a sequence $\left\{Q^{t}\right\}_{t=1}^{T}$ of blocking coalitions, Lemma C.14 enables us to define the -possibly empty- accessible subset of core $C_{a}\left(N, v,\left\{Q^{t}\right\}_{t=1}^{T}\right) \subseteq C(N, v)$ satisfying the conditions of Lemma C.14. By this lemma for any $c^{\prime} \in C_{a}\left(N, v,\left\{Q^{t}\right\}_{t=1}^{T}\right)$ we can generate a sequence $\left\{y^{t}\right\}_{t=1}^{T}$ of the required type. By Theorem 3.3 there exists a $\left\{\tilde{Q}^{t}\right\}_{t=1}^{T^{*}}$, such that the set $C_{a}\left(N, v,\left\{\tilde{Q}^{t}\right\}_{t=1}^{T^{*}}\right)$ is not empty, since there exists $c^{*} \in C_{a}\left(N, v,\left\{\tilde{Q}^{t}\right\}_{t=1}^{T^{*}}\right)$. Then our algorithm is as follows:

(1) Generate all coalition sequences of length at most $M$.

(2) For all sequences of blocking coalitions $\left\{Q^{t}\right\}_{y=1}^{T}$ do the following loop:

(a) Generate the set $C_{a}=C_{a}\left(N, v,\left\{Q^{t}\right\}_{t=1}^{T}\right)$.

(b) If $C_{a}$ is not empty exit loop.

(c) Take the next sequence.

(3) Generate $\left\{y^{t}\right\}_{t=1}^{T}$.

(4) Stop.

By Lemmata 3.4 and C.14 the algorithm terminates and produces a path with the required properties. By construction the algorithm is primitive recursive.

University of Maastricht, Department of Economics, P.O.Box 616, 6200 MD MaAsTRICHT, The NetherLANDS 